\title{
Propagating spin waves excited by spin-transfer torque: A combined electrical and optical study
}

\author{
M. Madami, ${ }^{1}$ E. Iacocca,,${ }^{2,3}$ S. Sani, ${ }^{4}$ G. Gubbiotti, ${ }^{5}$ S. Tacchi, ${ }^{5}$ R. K. Dumas, ${ }^{2}$ J. Åkerman, ${ }^{2,4}$ and G. Carlotti ${ }^{1}$ \\ ${ }^{1}$ Physics Department, University of Perugia, Perugia, Italy \\ ${ }^{2}$ Physics Department, University of Gothenburg, Gothenburg, Sweden \\ ${ }^{3}$ Department of Applied Physics, Division for Condensed Matter Theory, Chalmers University of Technology, Gothenburg, Sweden \\ ${ }^{4}$ Materials Physics, School of Information and Communications Technology, Royal Institute of Technology (KTH), Kista, Sweden \\ ${ }^{5}$ Istituto Officina dei Materiali del Consiglio Nazionale delle Ricerche (IOM-CNR), \\ Unità di Perugia clo Dipartimento di Fisica, Perugia, Italy
}

(Received 13 April 2015; revised manuscript received 9 June 2015; published 2 July 2015)

\begin{abstract}
Nanocontact spin-torque oscillators are devices in which the generation of propagating spin waves can be sustained by spin transfer torque. In the present paper, we perform combined electrical and optical measurements in a single experimental setup to systematically investigate the excitation of spin waves by a nanocontact spin-torque oscillator and their propagation in a $\mathrm{Ni}_{80} \mathrm{Fe}_{20}$ extended layer. By using microfocused Brillouin light scattering we observe an anisotropic emission of spin waves, due to the broken symmetry imposed by the inhomogeneous Oersted field generated by the injected current. In particular, spin waves propagate on the side of the nanocontact where the Oersted field and the in-plane component of the applied magnetic field are antiparallel, while propagation is inhibited on the opposite side. Moreover, propagating spin waves are efficiently excited only in a limited frequency range corresponding to wavevectors inversely proportional to the size of the nanocontact. This frequency range obeys the dispersion relation for exchange-dominated spin waves in the far field, as confirmed by micromagnetic simulations of similar devices. The present results have direct consequences for spin wave based applications, such as synchronization, computation, and magnonics.
\end{abstract}

DOI: 10.1103/PhysRevB.92.024403

PACS number(s): 85.75.-d, 75.30.Ds, 81.07.Lk, 78.35.+c

\section{INTRODUCTION}

Nanocontact (NC)-based spin-torque oscillators (STOs) [1] have been extensively studied in recent years due to their potential applications in a variety of fields, including spintronics, magnonics [2], and data storage [3]. Nanocontactbased spin-torque oscillators typically consist of an extended pseudo-spin-valve where two magnetic layers are separated by a nonmagnetic spacer. One of the magnetic layers is a hard magnet that acts as a spin polarizer and a reference layer, and it is referred to as the "fixed" layer. The second "free," magnetic layer is softer and thinner, making it susceptible to spintransfer torque (STT) induced dynamics [4,5]. Depending on the material used for the free layer, unique magnetodynamical modes can be generated in NC-STOs. On the one hand, by using $\mathrm{Co} / \mathrm{Ni}$ multilayers exhibiting perpendicular magnetic anisotropy, NC-STOs have been shown to support steady magnetization dynamics at low external fields [6] and even the excitation of localized modes known as magnetic dissipative droplets [7-10]. On the other hand, using soft magnets such as $\mathrm{NiFe}$, the existence of propagating [11,12], localized [12-15], and vortex $[16,17]$ modes has been demonstrated, depending on both the strength and the direction of the external magnetic field.

The propagating mode was predicted theoretically by Slonczewski [18] for a perpendicularly magnetized NC-STO, i.e., by applying an external magnetic field perpendicular to the device plane strong enough to saturate the free layer. This mode has been shown to consist of exchange-dominated spin waves (SWs) propagating radially away from the NC region at frequencies above the ferromagnetic resonance (FMR) frequency with a wavenumber inversely proportional to the $\mathrm{NC}$ diameter. The propagating nature of this mode lends itself to coupling NC-STOs to achieve better spectral features by synchronization [19-23], to perform computation [24,25], or to propagate information in magnonic devices [26,27].

The existence of the propagating mode was demonstrated only recently by employing either electrical measurements $[28,29]$, to study SW-mediated synchronization, or microfocused Brillouin light scattering ( $\mu$-BLS) [11], to directly detect the emitted SWs. Although these experiments provided unambiguous proof of the propagating character of this mode, micromagnetic simulations including the Oersted field generated by the bias current $[13,30]$ predicted that the propagation is generally anisotropic, especially if the applied field is tilted away from the device normal. This has tremendous consequences for the design and application of devices utilizing STT-driven SWs. However, to the best of our knowledge, no direct experimental evidence of such asymmetric emission has been provided to date for out-ofplane magnetized NC-STOs with well-defined propagating spin waves. Evidence of asymmetric emission has been experimentally demonstrated only for in-plane magnetized NC-STOs by the studies of Demidov et al. $[15,31]$ to date.

In the present paper, we combine electrical characterization and $\mu$-BLS in a single experimental setup to systematically investigate both the electrical properties of the STT-induced dynamics and the characteristics of the propagating SWs excited in an out-of-plane magnetized NC-STO. Evidence is provided for anisotropic SW propagation in these devices, as reproduced by micromagnetic simulations. More importantly, the STT-generated SWs are found to propagate within a wavevector range dictated by both the SW dispersion in the far field and the diameter of the NC. In fact, micromagnetic simulations reveal that nonlinear dynamics are strongly damped close to the NC (near-field region), while only wavevectors obeying the SW dispersion relation are able to propagate in the far-field region, i.e., at distances larger than the NC 
dimensions. These results are relevant for the development of SW-based devices, including the synchronization of NC-STO arrays, computation, and magnonics.

\section{A. Experimental details}

Samples were fabricated on a $\mathrm{SiO}_{2}(1 \mu \mathrm{m})$ covered silicon wafer through the following processing steps: a pseudo-spin-valve stack of $\mathrm{Pd}(8 \mathrm{~nm}) / \mathrm{Cu}(15 \mathrm{~nm}) / \mathrm{Co}(8 \mathrm{~nm}) /$ $\mathrm{Cu}(8 \mathrm{~nm}) / \mathrm{Ni}_{80} \mathrm{Fe}_{20}(4.5 \mathrm{~nm}) / \mathrm{Cu}(3 \mathrm{~nm}) / \mathrm{Pd}(3 \mathrm{~nm})$ was deposited by magnetron sputtering; then, making use of photolithography, rectangular $8 \times 16-\mu \mathrm{m}^{2}$ mesas were defined. A $30 \mathrm{~nm}$ thick $\mathrm{SiO}_{2}$ layer was deposited on top of the mesas to realize electric insulation of the devices. Defined in this insulation layer above each mesa were $2 \times 4 \mu \mathrm{m}^{2}$ ground electrodes and circular NCs with 100-nm diameter. Finally, contact pads with ground-signal-ground (GSG) geometry, as depicted in Fig. 1(c), were fabricated with sputter deposition of $1 \mu \mathrm{m} \mathrm{Cu} / 20 \mathrm{~nm}$ Au in a photolithography prepared lift-off pattern. The final device allows for $\mu$-BLS optical access on one side of the NC only, as shown in Fig. 1(c).

Measurements in the frequency domain were performed using a broadband spectrum analyzer (SA). A dc was injected into the $\mathrm{NC}$ while the device impedance was continuously monitored in order to avoid damage due to Joule heating. The direction of the injected $\mathrm{dc}$ is negative (i.e., electrons drift from the free to the fixed layer) as required to obtain an STT-induced enhancement of the magnetization dynamics in the free layer [11]. Notably, this approach is only sensitive to the magnetodynamics generated very near the NC, along the path of the dc.

Microfocused Brillouin light scattering measurements were performed applying an external magnetic field $H$ at an angle of about $15^{\circ}$ from the sample normal by means of a customized projected field electromagnet. As has already been demonstrated in a previous paper [11], the out-of-plane magnetized NC geometry favors the emission of propagating $\mathrm{SW}$ in the extended portion of the NiFe free layer. At the same time, the slight tilt of the external field allows us to define the direction of the in-plane component of the field, $H_{\mathrm{IP}}$, as shown in Fig. 1. This choice is crucial in defining the direction of the propagating SWs, as we will demonstrate below. The emitted SWs were experimentally detected at room temperature by means of a $\mu$-BLS setup described in detail elsewhere [32]. We measured the Stokes side of each spectrum only, in order to halve the total acquisition time. This approach is justified by the symmetry of the Stokes and anti-Stokes sides of the spectrum in a measurement performed at normal incidence in a thin magnetic film [33].

To summarize, our setup allows us to electrically investigate the generated dynamics of the NC-STO while simultaneously characterizing the emitted SWs by $\mu$-BLS.

\section{B. Micromagnetic simulations}

Micromagnetic simulations were performed using the graphic processing unit (GPU) accelerated software Mumax3 [34]. The extended free layer was modeled by a disk with a diameter of $2.2 \mu \mathrm{m}$ and absorbing boundary conditions (ABCs) to prevent spurious generation and reflection of SWs. The
ABCs were implemented by linearly increasing the Gilbert damping coefficient over $200 \mathrm{~nm}$ towards the disk edge, so that the active area of the model was a $1.8 \mu \mathrm{m}$ diameter disk. We considered the device nominal free layer thickness to be $4.5 \mathrm{~nm}$ and the magnetic parameters as follows: saturation magnetization $\mu_{o} M_{S}=0.69 \mathrm{~T}$, Gilbert damping $\alpha=0.01$, exchange stiffness $A=11 \mathrm{pJ} / \mathrm{m}$, and no magnetocrystalline anisotropy. These parameters define the cell discretization to $4.3 \times 4.3 \times 4.5 \mathrm{~nm}^{3}$, below the exchange length $\lambda_{\text {ex }} \approx 6 \mathrm{~nm}$, resulting in a $512 \times 512$ mesh. Spin-transfer torque was considered only in a cylindrical region defined by the $\mathrm{NC}$ of diameter $d=100 \mathrm{~nm}$ positioned in the geometrical center of the disk. We assumed a polarization consistent with a Co layer, $P=0.3$, and a symmetric torque $\lambda=1$, as has previously been shown to model similar devices with high accuracy $[13,14]$. The direction of the fixed layer was calculated numerically by solving the magnetostatic boundary conditions for a $\mathrm{Co}$ thin film with saturation magnetization $\mu_{o} M_{P}=1.5 \mathrm{~T}$. Both the external applied field and the current-generated Oersted field were included in the simulations. Unless specified, the simulations were performed with a fixed time step of $10 \mathrm{fs}$ at room temperature.

The spectral characteristics of the generated dynamics can be estimated from $10 \mathrm{~ns}$ long simulations sampled at $10 \mathrm{ps}$, returning a frequency resolution of $\approx 97 \mathrm{MHz}$. Field-dependent simulations indicate that high field magnitudes are required to excite well-defined SWs. This can be understood from the fact that demagnetizing fields arise at the boundaries of the simulated disk, favoring a vortex ground state. Applied fields of magnitude $\mu_{o} H>650 \mathrm{mT}$ are numerically found to preclude the formation of a vortex and to allow the excitation of STTinduced propagating SWs.

\section{RESULTS AND DISCUSSION}

\section{A. Anisotropic spin-wave emission and decay length}

The first step of our experiment was to investigate the effect of varying the direction of the in-plane component of the external magnetic field $\left(H_{\mathrm{IP}}\right)$ on the spatial distribution of SWs emitted below the NC area. To perform such an experiment the external field intensity was set to $\mu_{o} H=+700 \mathrm{mT}$ and the field direction was tilted so that its in-plane component was antiparallel to the Oersted field on the side of the NC which is accessible to $\mu$-BLS, as shown in Fig. 1(a). By injecting a dc of $I=30 \mathrm{~mA}$ into the $\mathrm{NC}$, we were able to obtain steady-state oscillations at a frequency of $15.5 \mathrm{GHz}$ with an intensity of about $+9 \mathrm{~dB}$ over noise, as measured by the SA. In order to map the intensity of the emitted SWs, we then performed a two-dimensional $\mu$-BLS scan over an area of about $2 \times 1 \mu \mathrm{m}^{2}$ with a step size of $250 \mathrm{~nm}$ on the optically accessible NC side at a distance of about $1 \mu \mathrm{m}$ from the NC itself. The measured two-dimensional map, shown in Fig. 1(a), was obtained by integrating the measured SW intensity in a frequency range of $0.5 \mathrm{GHz}$ around the central value of $15.5 \mathrm{GHz}$. This $\mu$-BLS map clearly shows emission from the $\mathrm{NC}$ area, confined in a relatively narrow beam which propagates in the direction perpendicular to both the direction of the Oersted field and $H_{\mathrm{IP}}$. If the external field polarity is reversed $\left(\mu_{o} H=-700 \mathrm{mT}\right)$, the Oersted field and $H_{\mathrm{IP}}$ become 


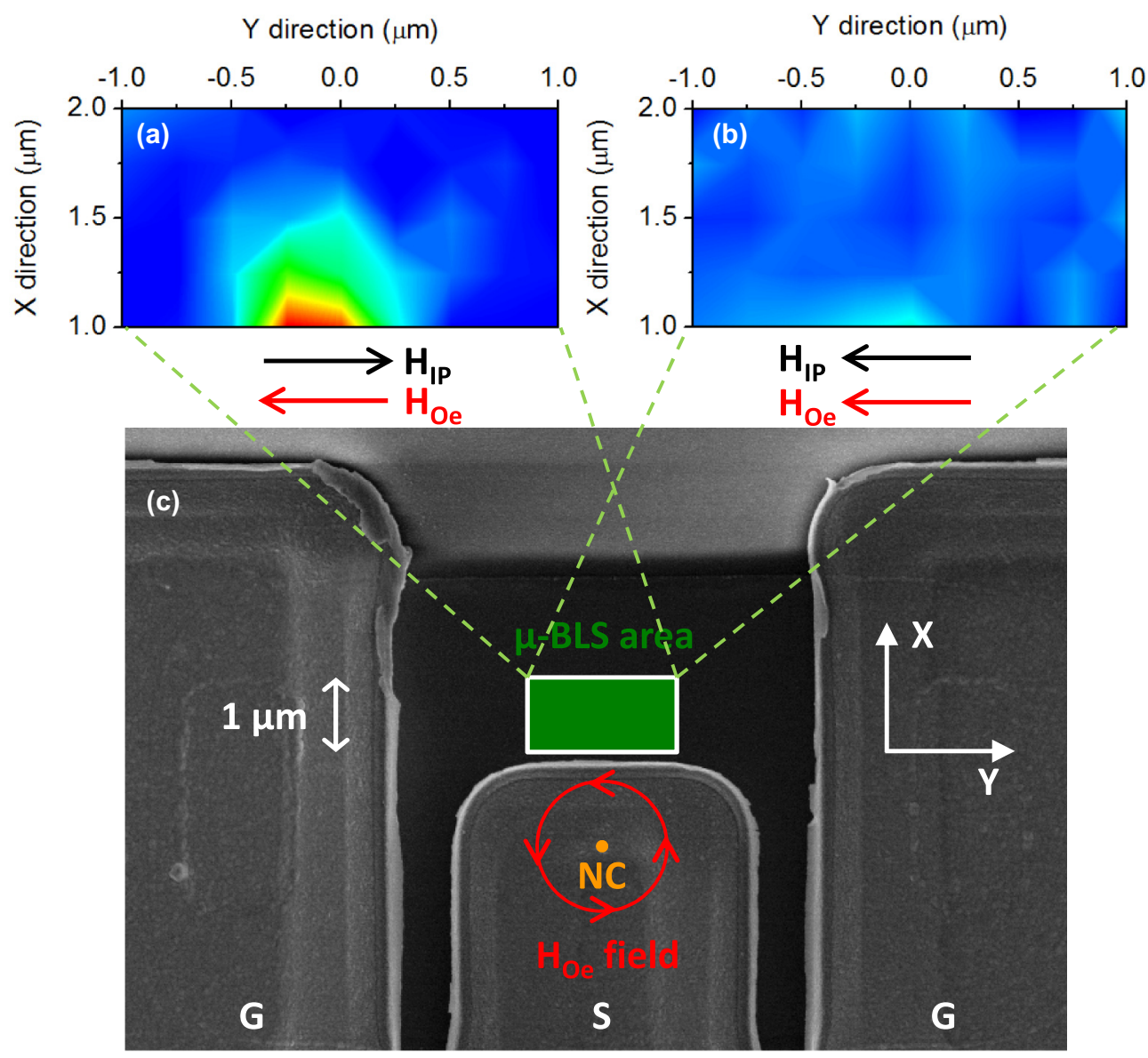

FIG. 1. (Color online) (a, b) SW intensity maps measured by $\mu$-BLS in the green area of panel (c) for two different directions of the in-plane component $\left(H_{\mathrm{IP}}\right)$ of the external field, $H=700 \mathrm{mT}$ and $I=30 \mathrm{~mA}$. (c) Scanning electron microscope (SEM) image of the device with the GSG pads, NC position and the direction of the Oersted (Oe) field generated by the current (I) flowing into the NC.

parallel on the optically accessible NC side [29] but no SWs are detected in this case, as shown by the $\mu$-BLS map in Fig. 1(b) [35]. This clearly demonstrates anisotropic SW emission, confirming previous numerical predictions indicating that the largest SW intensity is emitted on the side of the NC where

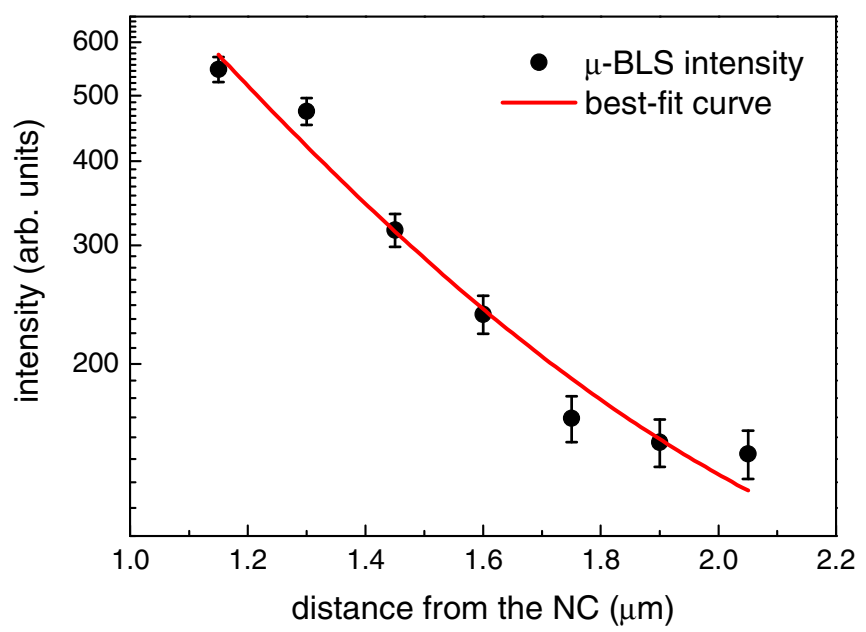

FIG. 2. (Color online) SW intensity (circles) measured by $\mu$-BLS as a function of the distance $\left(X-X_{0}\right)$ from the NC position. Best-fit curve (line) obtained using Eq. (1). the Oersted and the $H_{\mathrm{IP}}$ fields are antiparallel [13,30]. As the second step of this investigation, we measured the decay of the emitted SW intensity $(i)$ as a function of the distance from the NC position $\left(X-X_{0}\right)$, by scanning the laser probe along the $X$ direction. The results of these measurements are shown in Fig. 2 (black dots) together with the result of a best fit analysis obtained using the following analytical expression:

$$
i(X)=i_{0}+\frac{A}{X-X_{0}} \cdot e^{-\frac{X-X_{0}}{l}}
$$

where $i_{0}$ is the baseline, $X_{0}$ is the NC position (fixed at $X_{0}=0$ ), and $l$ is the decay length of SW intensity; $A$ and $l$ are the free parameters in the fit routine, which returns a value of $l=500 \pm 50 \mathrm{~nm}$. This is in very good agreement with the expected value obtained from the expression $l=v_{g} / 2 \alpha \omega=500 \mathrm{~nm}$, where $v_{g}=1.0 \mu \mathrm{m} / \mathrm{ns}$ is the $\mathrm{SW}$ group velocity extracted from the simulated dispersion curve of the free layer (see Fig. 5), $\alpha=0.01$ is the conventional value of damping in $\mathrm{NiFe}$, and $\omega=2 \pi f$ is the $\mathrm{SW}$ angular frequency $(f=15.5 \mathrm{GHz})$.

\section{B. Dependence of the spin-wave emission on the injected current and applied field intensity}

Next, we turn our attention to the characteristics of the emitted SWs by simultaneously measuring the spectra 
(a)

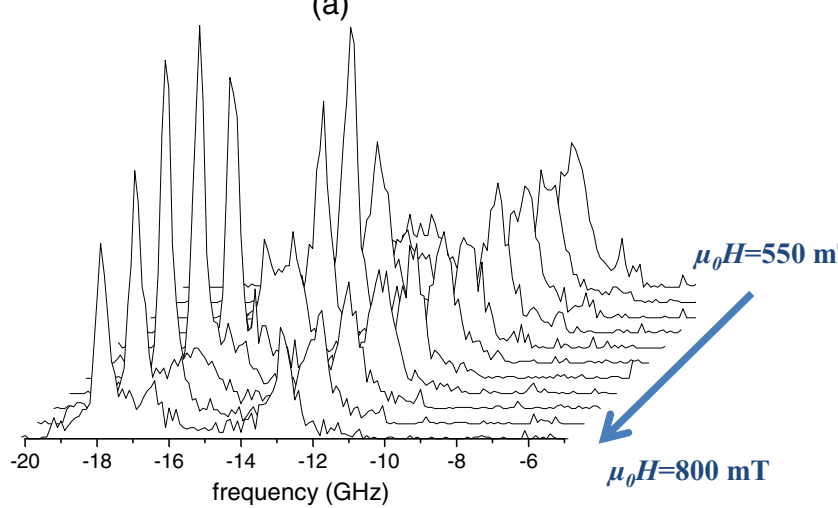

(b)



(c)



(d)

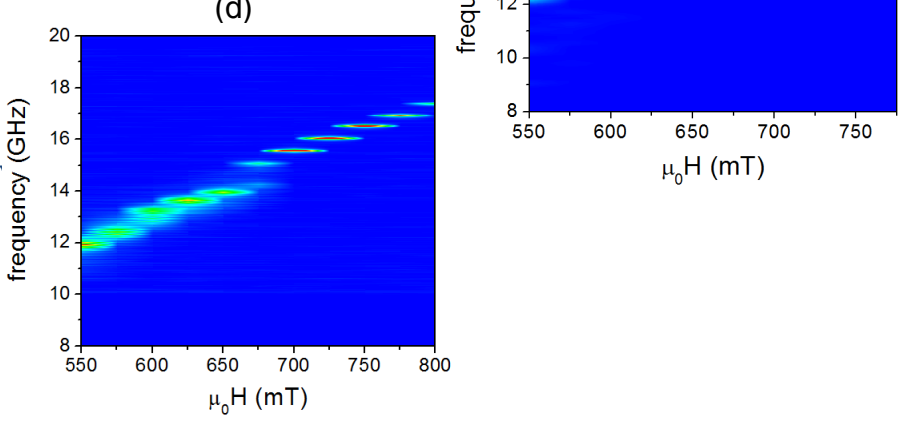

(e)

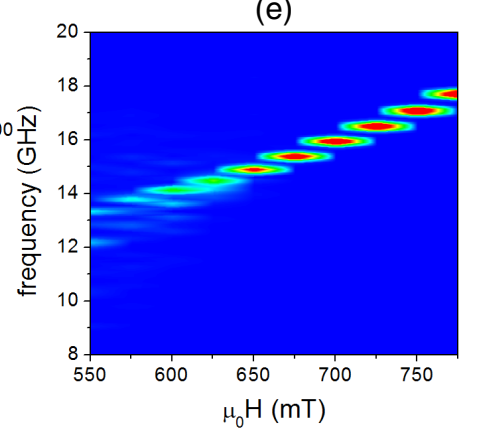

FIG. 3. (Color online) (a) $\mu$-BLS and (b) SA spectra measured as a function of the intensity of the external field $H$ for a fixed value of the injected dc $I=30 \mathrm{~mA}$. (c, b) Color plots of the sequence of spectra in panels (a) and (b). (e) Results of micromagnetic simulations obtained under the same conditions.

obtained electrically and optically when varying either the intensity of the external magnetic field $H$ or the direct current I. The results reported in Fig. 3 were obtained by injecting a constant current $I=30 \mathrm{~mA}$ and applying an external magnetic field of variable intensity (in the range $H=550-800 \mathrm{mT}$ ) with its in-plane component $\left(H_{\mathbf{I P}}\right)$ directed as in Fig. 1(a), to favor the emission of SWs on the optically accessible side of the device. The first two panels show the $\mu$-BLS spectra of SW intensity measured at a fixed position of about $1.0 \mu \mathrm{m}$ away from the NC [Fig. 3(a)] and the sequence of spectra acquired with the SA [Fig. 3(b)] as a function of $H$. The same data are reported in Figs. 3(c) and 3(d) on color scale bidimensional plots. The data obtained from $\mu$-BLS features two well-defined peaks. The lowest frequency peak, which is not observed on the SA spectra [Fig. 3(d)], exhibits a constant intensity and can be measured even for $I=0$ [cf. red spectrum in Fig. 4(a)]. It corresponds to the thermally activated FMR mode of the NiFe layer, consistent with Kittel's equation as a function of $H$. The highest frequency peak of Fig. 3(c) is instead observed in the SA spectra at the same frequency for all the measured fields and corresponds to the SWs emitted by the STT-driven precession of the magnetization under the NC. The blue-shift of this peak with respect to the FMR frequency is a signature of the propagating character of the SWs emitted away from the NC, which is further confirmed by the fact that its signal is measurable up to about $2.2 \mu \mathrm{m}$ away from the NC itself, as was shown in Fig. 2. The positive field tunability of this mode is estimated to be $24 \mathrm{MHz} / \mathrm{mT}$. A comparison of the above experimental results with those obtained from micromagnetic simulations [Fig. 3(e)] accounts for a very good quantitative agreement over the entire range of fields we investigated. Careful inspection of the measured spectra reveals the presence of a mode transition for a field value of $H=675 \mathrm{mT}$, denoted by two resonant frequencies in both the $\mu$-BLS and SA spectra. Corresponding to this mode transition, the SA spectrum is strongly modified, exhibiting a sharper and more intense peak. The occurrence of such mode transitions is very common in this kind of NC-STO devices $[28,29]$ and reflects the complicated nonlinear dynamics that can also be affected by unique and local features of the real NC. As a matter of fact, nominally identical devices present one or more mode transitions at different field/current values, or even no transitions at all.

A direct comparison of the measured peaks corresponding to the FMR and STT modes in the $\mu$-BLS spectra [Fig. 3(a)] suggests that the two modes have comparable intensities. This result may seem surprising at first, since a much larger intensity should be expected for STT-driven SWs than for thermally excited SWs. The reason for this apparent inconsistency lies in the wavevector content of the two modes and how it is efficiently collected by the optics of our experimental setup. By combining the effect of the finite collection angle $\left[\theta=48.6^{\circ}, \mathrm{NA}=\sin (\theta)=0.75\right]$ of our microscope objective with the effect of the uncertainty in the in-plane component of the wavevector of the scattered photons, resulting from the limited spatial extent of the laser spot on the sample $(\approx 300 \mathrm{~nm})$, it is possible to demonstrate that the efficiency of our apparatus in detecting SWs with small wavelengths 


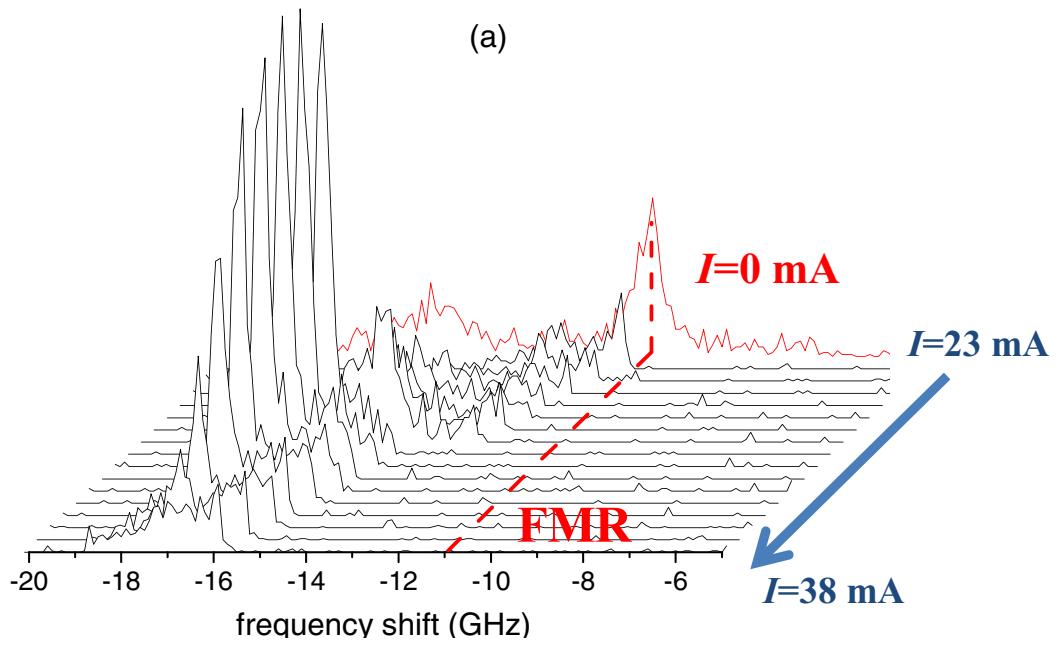

(b)

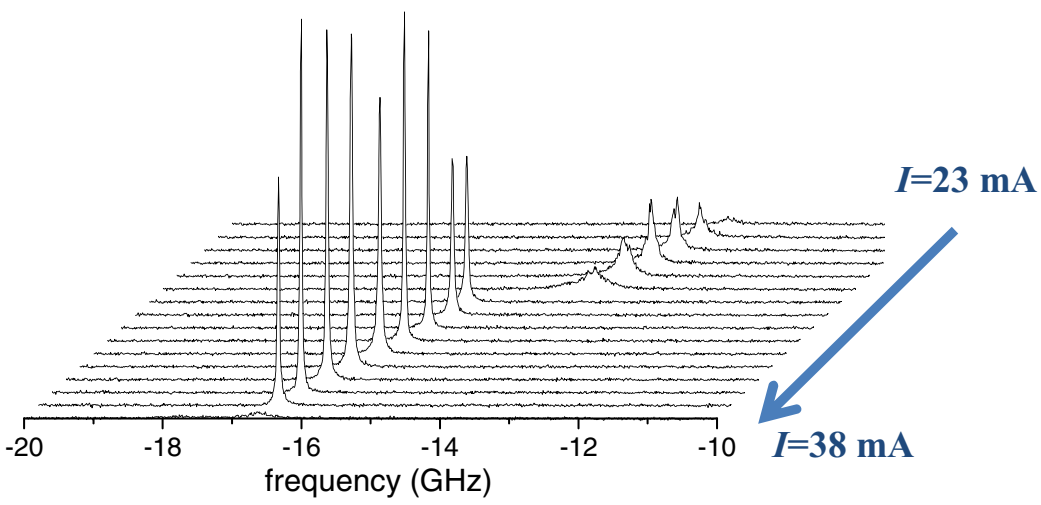

(c)

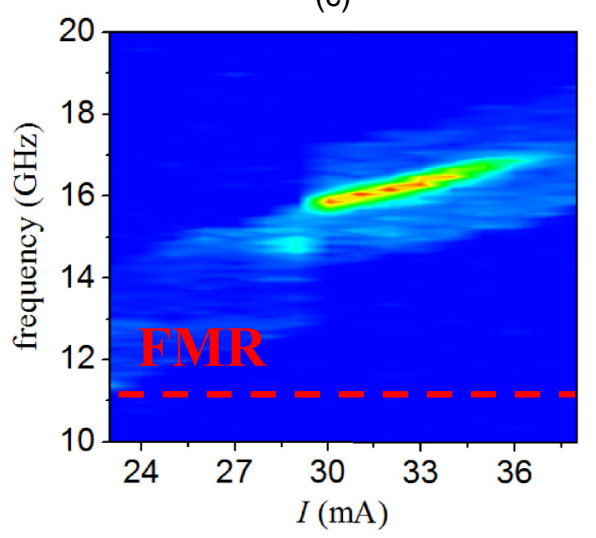

(d)

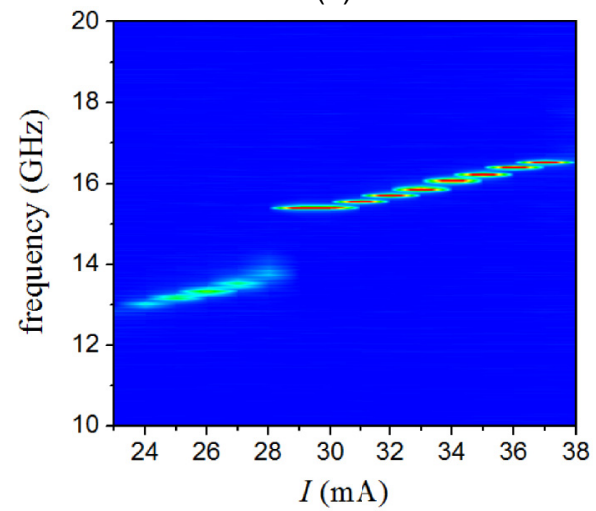

FIG. 4. (Color online) (a) $\mu$-BLS and (b) SA spectra measured as a function of the intensity of the injected dc $(I)$ for a fixed value of the external field $H=680 \mathrm{mT}$. (c, b) Color plots of the sequence of spectra in panels (a) and (b).

decreases very rapidly as the wavelength is reduced below $300 \mathrm{~nm}$ [11]. Since the diameter of the NC under investigation is only $100 \mathrm{~nm}$, we expect the wavelength of propagating SWs to be smaller than $200 \mathrm{~nm}$ [18], which means we can detect them with relatively low efficiency in our apparatus.

Figure 4 shows the results of a second set of simultaneous $\mu$-BLS and SA characterizations obtained by setting the external field intensity to a fixed value of $H=680 \mathrm{mT}$ and varying the intensity of the injected $\mathrm{dc}(I)$ over the range between 23.0 and $38.0 \mathrm{~mA}$. The first $\mu$-BLS spectrum (red line) in Fig. 4(a) was measured at $I=0 \mathrm{~mA}$, showing the thermal FMR mode. In the following sequence of $\mu$-BLS spectra [Fig. 4(a)], the FMR signal was no longer acquired, in order to reduce the acquisition time and because its frequency does not vary with current, so the only visible peak in the spectra is the one corresponding to the STT-driven SW excitation. Figure 4(b) shows the corresponding sequence of SA spectra, while Figs. 4(c) and 4(d) report the same $\mu$-BLS and SA measurements in bidimensional color scale plots. Similarly, as in the case of the field characterization (Fig. 3), we observed a blue-shift of the STT-driven SW frequencies with respect to the FMR, as well as a positive current tunability that we estimate to be $250 \mathrm{MHz} / \mathrm{mA}$. On increasing $I$, a mode transition is observed for the value $I=29.0 \mathrm{~mA}$; this is characterized by a relatively large frequency jump of about $2 \mathrm{GHz}$, clearly visible in the $\mathrm{SA}$ sequence of spectra, and accompanied by a dramatic reduction in linewidth and an increase in maximum intensity. It is interesting to note how the signal coming from propagating SWs, as measured by $\mu$-BLS, is easily detectable only over a finite range of currents, $I=29.0-35.0 \mathrm{~mA}$, after the mode transition. Before the mode transition $(I<29.0 \mathrm{~mA})$ the SW signal is hardly detectable, probably because of the very low STT efficiency, as is confirmed by the electric measurements. More interesting is the existence of an upper bound $(I=35.0 \mathrm{~mA})$, above which the intensity of the emitted SWs rapidly decreases. This is discussed in greater detail in the following paragraphs.

The existence of a well-defined $\mathrm{dc}$ range (and a corresponding frequency range), in which propagating SWs are experimentally detected, is a reproducible feature that we observed in several NC-STO devices with slightly different NC diameters, in the range $80-120 \mathrm{~nm}$, obtaining results which are in good qualitative agreement with those presented in this paper. In order to shed more light on this effect, we calculated the dispersion curve for SWs in the out-of-plane magnetized $\mathrm{NiFe}$ layer $(H=680 \mathrm{mT})$. The resulting frequency vs $1 / \lambda$ plot is shown in Fig. 5(b), where it is compared with the frequency vs $I$ plot of 


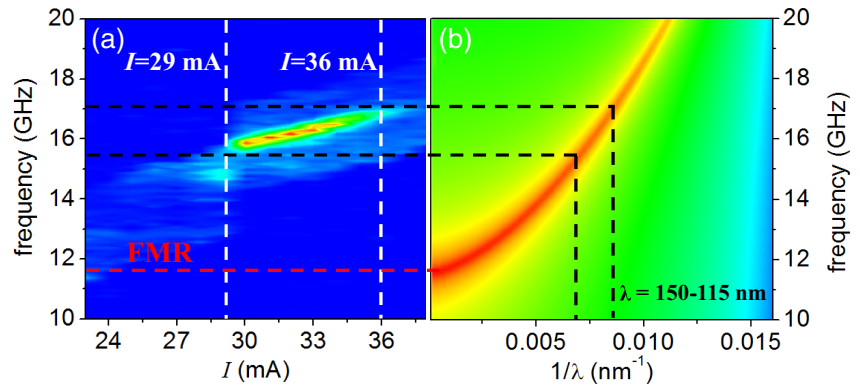

FIG. 5. (Color online) (a) $\mu$-BLS spectra measured as a function of the intensity of the injected dc $(I)$ for a fixed value of the external field $H=680 \mathrm{mT}$. (b) Simulated SW dispersion curve of the NiFe free layer.

Fig. 5(a). We can see good quantitative agreement between the frequency of the FMR mode, as measured by $\mu$-BLS, and the simulated frequency at $1 / \lambda=0(k=2 \pi / \lambda=0)$. From a direct comparison between the two panels in Fig. 5, it is clear that the finite frequency range detected experimentally corresponds to a finite wavelength range of $\lambda=115-150 \mathrm{~nm}$. This is the central result of the present paper, as it suggests that propagating SWs emitted by an out-of-plane magnetized NC-STO have a limited range of accessible wavelengths, which is ultimately associated with the NC diameter and the exchange-dominated dispersion relation of SWs [36]. In other words, a NC-STO cannot efficiently excite exchangedominated SWs with a wavelength much shorter (or longer) than its own NC diameter. This has tremendous implications for the synchronization of NC-STOs, since the wavelength unambiguously determines the phase difference between the devices and ultimately the phase-locking condition.

The above observations can be further tested using micromagnetic simulations. Due to the finite size of the simulated disk, much stronger fields are required to obtain a coherent SW emission. Consequently, we set a field of magnitude $H=800 \mathrm{mT}$ tilted $15^{\circ}$ from the plane normal in the following simulations. To characterize the allowed propagating SWs, we performed simulations at $T=0 \mathrm{~K}$ and determined their wavelength as a function of distance via smoothed pseudoWigner-Ville (SPWV) transform. By this method, it is possible to tune the real and reciprocal space resolution by making use of smoothing windows. The wavelength content of the generated SW is determined by performing a SPWV transform on a simulation snapshot along the $X$ direction, where we used a Gaussian window of $4.3 \mathrm{~nm}$ for the real space and a Hann window of $1.9 \mu \mathrm{m}^{-1}$ for the reciprocal space. We then averaged 100 SPWV transforms, each one calculated from different snapshots of the same time-dependent simulation, to further improve the accuracy of the method. The results for NC-STO driven at 34 and $25 \mathrm{~mA}$ are shown as color plots in Figs. 6(a) and 6(b), respectively, where the vertical black lines indicate the boundaries of the NC and the color represents the normalized SW magnitude in logarithmic scale. The choice of the values for $I$ was made in order to have one value below the SW emission threshold $(25 \mathrm{~mA})$ and the other one right in the middle of that range $(34 \mathrm{~mA})$, as confirmed by Fig. 5(a). We remind the reader that this figure must be understood as the wavelength content ( $y$ axis) as a function of distance for the SW propagating along $X$. Whether the

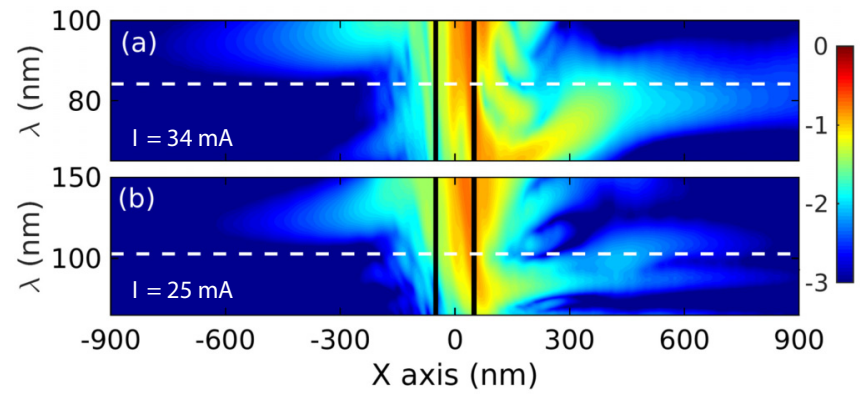

FIG. 6. (Color online) SPWV transform of simulated SWs along $X$ for (a) $I=34 \mathrm{~mA}$ and (b) $I=25 \mathrm{~mA}$ and an external field of $H=800 \mathrm{mT}$. The vertical black lines indicate the boundaries of the $\mathrm{NC}$ while the white dashed lines indicate wavelength obtained from the SW dispersion relation at each frequency, namely (a) $19.2 \mathrm{GHz}$ and (b) $17.1 \mathrm{GHz}$. The color contrast indicates the normalized magnitude in logarithmic scale of the SW associated with each wavelength ( $y$ axis) as a function of $X$.

NC-STO is driven at $34 \mathrm{~mA}$ [Fig. 6(a)] or $25 \mathrm{~mA}$ [Fig. 6(b)], most of the wavelength content is rapidly damped within about $0.2 \mu \mathrm{m}$ from the NC center (near-field region). This strong wavelength content describes the nonlinear forced dynamics of the system due to the presence of strong/local effects in the NC area such as the STT effect and the Oersted field. However, when the NC is driven at $34 \mathrm{~mA}$ [Fig. 6(a)], a band of wavelengths is observed to prevail up to the simulation boundary, and only in the $+X$ direction. This wavelength band is in good quantitative agreement with the wavelength analytically obtained from the $\mathrm{SW}$ dispersion relation for $\mathrm{NiFe}$ at the driven NC-STO frequency (white dashed line) and similar to the NC diameter. In contrast, when the NC-STO is driven at $25 \mathrm{~mA}$ [Fig. 6(b)], this wavelength band, although present, is rapidly damped within about $0.6 \mu \mathrm{m}$, corresponding to a scenario in which the Oersted field strongly distorts the magnetic landscape. It is worth mentioning that these results indicate that $\mathrm{SW}$ do propagate in the $-X$ direction as well, as expected from exchange coupling, but their energy is negligible for distances greater than $0.5 \mu \mathrm{m}$ from the NC. An additional interesting feature of Fig. 6 is that the wavelengths close to the $\mathrm{NC}$ boundary are lower (higher) in the $+X(-X)$ direction corresponding to the decrease (increase) of the local field magnitude induced by the Oersted field.

\section{CONCLUSIONS}

The properties of propagating SWs emitted by an out-ofplane magnetized NC-STO were investigated by means of a combined radio frequency (RF) and $\mu$-BLS experimental setup. We experimentally demonstrated the anisotropic emission of SWs, which is concentrated on the side of the $\mathrm{NC}$ where the Oersted field and the in-plane component of the external field are antiparallel. The analysis of both the field and the current tunability of the device showed a clear blue-shift of the STT-excited SW frequency with respect to that of the FMR, as well as the presence of abrupt mode transitions. More importantly, it was found that propagating SWs are only efficiently excited over a limited interval of wavelengths comparable with the NC diameter. Given the dispersion relation in the far field, this corresponds to a range 
of frequencies of a few gigahertz. We believe these results will be of the utmost importance for further progress in NC-STO synchronization, computation, and magnonic applications via emitted SWs.

\section{ACKNOWLEDGMENTS}

Support from the European Community's Seventh Framework Programme (FP7/2007-2013) under Grant No. 318287
"LANDAUER" and by the Ministero Italiano dell'Università e della Ricerca (MIUR) under the PRIN2010 project (No. 2010ECA8P3) is gratefully acknowledged. Support from the Swedish Research council (VR), the Swedish Foundation for Strategic Research (SSF), and the Knut and Alice Wallenberg Foundation is gratefully acknowledged. E. Iacocca acknowledges support from the Swedish Research Council, Reg. No. 637-2014-6863.
[1] T. Silva and W. Rippard, J. Magn. Magn. Mater. 320, 1260 (2008).

[2] R. L. Stamps, S. Breitkreutz, J. Åkerman, A. V. Chumak, Y. Otani, G. E. W. Bauer, J.-U. Thiele, M. Bowen, S. A. Majetich, M. Kläui, I. L. Prejbeanu, B. Dieny, N. M. Dempsey, and B. Hillebrands, J. Phys. D: Appl. Phys. 47, 333001 (2014).

[3] J. Åkerman, Science 308, 508 (2005).

[4] J. Slonczewski, J. Magn. Magn. Mater. 159, L1 (1996).

[5] L. Berger, Phys. Rev. B 54, 9353 (1996).

[6] W. Rippard, A. Deac, M. Pufall, J. Shaw, M. Keller, S. Russek, G. Bauer, and C. Serpico, Phys. Rev. B 81, 014426 (2010).

[7] M. Hoefer, T. Silva, and M. Keller, Phys. Rev. B 82, 054432 (2010).

[8] S. Mohseni, S. Sani, J. Persson, T. Nguyen, S. Chung, Y. Pogoryelov, P. Muduli, E. Iacocca, A. Eklund, R. Dumas, S. Bonetti, A. Deac, M. Hoefer, and J. Åkerman, Science 339, 1295 (2013).

[9] E. Iacocca, R. Dumas, L. Bookman, M. Mohseni, S. Chung, M. Hoefer, and J. Åkerman, Phys. Rev. Lett. 112, 047201 (2014).

[10] F. Macià, D. Backes, and A. D. Kent, Nat. Nanotechnol. 9, 992 (2014).

[11] M. Madami, S. Bonetti, G. Consolo, S. Tacchi, G. Carlotti, G. Gubbiotti, F. Mancoff, M. Yar, and J. Åkerman, Nat. Nanotechnol. 6, 635 (2011).

[12] S. Bonetti, V. Tiberkevich, G. Consolo, G. Finocchio, P. Muduli, F. Mancoff, A. Slavin, and J. Åkerman, Phys. Rev. Lett. 105, 217204 (2010).

[13] R. Dumas, E. Iacocca, S. Bonetti, S. Sani, S. Mohseni, A. Eklund, J. H. O. Persson, and J. Åkerman, Phys. Rev. Lett. 110, 257202 (2013).

[14] E. Iacocca, P. Dürrenfeld, O. Heinonen, J. Åkerman, and R. Dumas, Phys. Rev. B 91, 104405 (2015).

[15] V. E. Demidov, S. Urazhdine, and S. O. Demokritov, Nat. Mater., 9, 984 (2010).

[16] T. Devolder, J.-V. Kim, M. Manfrini, W. van Roy, L. Lagae, and C. Chappert, Appl. Phys. Lett. 97, 072512 (2010).

[17] S. Sani, J. Persson, S. Mohseni, V. Fallahi, and J. Åkerman, J. Appl. Phys. 109, 07C913 (2011).

[18] J. Slonczewski, J. Magn. Magn. Mater. 195, L261 (1999).

[19] S. A. Kaka, M. R. Pufall, W. H. Rippard, T. J. Silva, S. E. Russek, and J. A. Katine, Nature 437, 389 (2005).

[20] F. Mancoff, N. Rizzo, B. Engel, and S. Tehrani, Nature 437, 393 (2005).
[21] S. Sani, J. Persson, S. Mohseni, Y. Pogoryelov, P. Muduli, A. Eklund, G. Malm, M. Käll, A. Dmitriev, and J. Åkerman, Nat. Commun. 4, 2731 (2013).

[22] A. Houshang, E. Iacocca, P. Dürrenfeld, J. Åkerman, and R. Dumas (unpublished).

[23] T. Kendziorczyk, S. Demokritov, and T. Kuhn, Phys. Rev. B 90, 054414 (2014).

[24] F. Macià, A. Kent, and F. Hoppensteadt, Nanotechnology 22, 095301 (2011).

[25] F. Macià, F. C. Hoppensteadt, and A. D. Kent, Nanotechnology 25, 045303 (2014).

[26] S. Bonetti and J. Åkerman, in Magnonics, edited by Sergej O. Demokritov and Andrei N. Slavin (Springer, Berlin, 2013), Chap. 13, pp. 177-187.

[27] S. Urazhdin, V. E. Demidov, H. Ulrichs, T. Kendziorczyk, T. Kuhn, J. Leuthold, G. Wilde, and S. O. Demokritov, Nat. Nanotechnol. 9, 509 (2014).

[28] M. Pufall, W. Rippard, S. Russek, S. Kaka, and J. Katine, Phys. Rev. Lett. 97, 087206 (2006).

[29] M. R. Pufall, W. H. Rippard, S. E. Russek, and E. R. Evarts, Phys. Rev. B 86, 094404 (2012).

[30] M. Hoefer, T. Silva, and M. Stiles, Phys. Rev. B 77, 144401 (2008).

[31] V. E. Demidov, S. Urazhdin, V. Tiberkevic, A. Slavin, and S. O. Demokritov, Phys. Rev. B 83, 060406 (2011).

[32] M. Madami, G. Gubbiotti, S. Tacchi, and G. Carlotti, in Solid State Physics, edited by Robert E. Camley and Robert L. Stamps (Academic Press, Burlington, MA, 2012), Vol. 63, Chap. 2, pp. 79-150.

[33] M. Madami, S. Tacchi, G. Gubbiotti, G. Carlotti, F. Montoncello, G. Capuzzo, and F. Nizzoli, J. Phys. Conf. Ser. 200, 042008 (2010).

[34] A. Vansteenkiste et al., arXiv:1406.7635.

[35] The frequency of the steady-state oscillations as measured by the SA only changed by a small amount (from 15.5 to $15.3 \mathrm{GHz}$ ) when the applied field was reversed, while its intensity increased to $+10 \mathrm{~dB}$ over noise.

[36] The analysis was performed only on the current-dependent, and not on the field-dependent, measurements, because if one modifies the external field, the whole SW dispersion curve would be modified for every given value of the field. This does not happen in the current-dependent measurements. As a consequence, it would be much more difficult to extract useful information from such a complex analysis. 